\title{
A generalized Rayleigh distribution and its application
}

\begin{abstract}
This paper studies properties and applications of the generalized Rayleigh-truncated negative binomial distribution established by Jiju \& Lishamol. ${ }^{1}$ For the concerned objective, we applied the model to a real life data set and its performance is compared with that of other four-parameter generalized Rayleigh distributions which are derived using different generators.
\end{abstract}

Keywords: hazard rate function, marshall-olkin family, maximum likelihood estimation, rayleigh-truncated negative binomial distribution
Volume 8 Issue 4 - 2019

\author{
Lishamol Tomy,' Jiju Gillariose ${ }^{2}$ \\ 'Department of Statistics, Deva Matha College, India \\ ${ }^{2}$ Department of Statistics, St.Thomas College, India
}

\begin{abstract}
Correspondence: Lishamol Tomy, Department of Statistics, Deva Matha College, Kuravilangad, Kerala-686633, India, Email lishatomy@gmail.com
\end{abstract}

Received: May 31, 2019 | Published: July 08, 2019

\section{Introduction}

The Rayleigh distribution was introduced by Rayleigh ${ }^{2}$ and originally proposed in the fields of acoustics and optics. It has emerged as a special case of the Weibull distribution. The Rayleigh distribution has widely used in communication theory to describe hourly median and instantaneous peak power of received radio signals. Moreover, It has received a considerable attention from engineers and physicists for modeling wave propagation, radiation, synthetic aperture radar images, and other related phenomena. There have been many forms for the Rayleigh distribution to provide flexibility for modeling data. $\operatorname{Vod}^{3,4}$ proposed a powerful extension of the Rayleigh distribution and studied its properties. Its probability density function (pdf) is given by

$$
\mathrm{f}(\mathrm{x}, \theta, \lambda)=\frac{2 \theta^{-2 \lambda-2} \mathrm{x}^{2 \lambda+1} \mathrm{e}^{-(\mathrm{x} / \theta)^{2}}}{\Gamma(\lambda+1)}, \mathrm{x}, \theta>0, \lambda>-1 .
$$

where $\theta$ is the scale parameter, $\lambda$ is the shape parameter and $\Gamma(\mathrm{a})=\int_{0}^{\infty} \mathrm{t}^{\mathrm{a}-1} \mathrm{e}^{-\mathrm{t}} \mathrm{dt}$ is the complete gamma function. Its survival function is

$$
\overline{\mathrm{F}}(\mathrm{x}, \theta, \lambda)=1-\frac{\Gamma\left(\lambda+1,(\mathrm{x} / \theta)^{2}\right)}{\Gamma(\lambda+1)}=\bar{\gamma}\left(\lambda+1,(\mathrm{x} / \theta)^{2}\right), \mathrm{x}, \theta>0, \lambda>-1 .
$$

where $\Gamma(\mathrm{a}, \mathrm{x})=\int_{0}^{\mathrm{x}} \mathrm{t}^{\mathrm{a}-1} \mathrm{e}^{-\mathrm{t}} \mathrm{dt}$ is the incomplete gamma function, hence statistical software's can be used for various values of $\theta$ and $\lambda$. In literature, there are many studies based on extensions of this generalized Rayleigh (GR) distribution using different generators. Cordeiro et al., ${ }^{5}$ derived four-parameter beta-GR distribution, Gomes et al., ${ }^{6}$ proposed the four-parameter Kumaraswamy-GR distribution, and MirMostafaee et al., ${ }^{7}$ introduced Marshall-Olkin extended GR distribution respectively.

In modern era, the literature has suggested several ways of extending well-known distributions to generate a more flexible of distributions. Recently new generator approach introduced by Nadarajah et al., ${ }^{8}$ as a generalization of the family of Marshall-Olkin extended (MOE) distributions by Marshall \& Olkin. ${ }^{9}$ This approach deals with the shape parameter induction in parent (or baseline) distribution to explore tail properties and to improve goodness-offits. Let $\mathrm{X}_{1}, \mathrm{X}_{2}, \ldots$ be a sequence of independent and identically distributed random variables with survival function $\overline{\mathrm{F}}(\mathrm{x})$ and $\mathrm{N}$ be a truncated negative binomial random variable, independent of $\mathrm{X}_{\mathrm{i}}$ 's, with parameters $0<\alpha<1$ and $\beta>0$, such that

$$
\mathrm{P}(\mathrm{N}=\mathrm{n})=\frac{\alpha^{\beta}}{1-\alpha^{\beta}}\left(\begin{array}{c}
\beta+\mathrm{n}-1 \\
\beta-1
\end{array}\right)(1-\alpha)^{\mathrm{n}} ; \mathrm{n}=1,2, \ldots
$$

If $\mathrm{U}_{\mathrm{N}}=\min \left(\mathrm{X}_{1}, \mathrm{X}_{2}, \ldots \mathrm{X}_{\mathrm{N}}\right)$, then the survival function of $\mathrm{U}_{\mathrm{N}}$ is

$$
\overline{\mathrm{G}}(\mathrm{x}, \alpha, \beta)=\frac{\alpha^{\beta}}{1-\alpha^{\beta}}\left\{[\mathrm{F}(\mathrm{x})+\alpha \overline{\mathrm{F}}(\mathrm{x})]^{-\beta}-1\right\} \mathrm{x} \in \mathrm{R}, \alpha, \beta>0
$$

Similarly, if $\alpha>1$ and $\mathrm{N}$ is a truncated negative binomial random variable with parameters $\frac{1}{\alpha}$ and $\beta>0$, then $\mathrm{W}_{\mathrm{N}}=\max \left(\mathrm{X}_{1}, \mathrm{X}_{2}, \ldots \mathrm{X}_{\mathrm{N}}\right)$ also has the same survival function given in (4). If $\alpha \rightarrow 1$ in (4), then $\overline{\mathrm{G}}(\mathrm{x}, \alpha, \beta) \rightarrow \overline{\mathrm{F}}(\mathrm{x})$. If $\beta=1$, then this family reduces to the Marshall-Olkin family of distributions. The pdf of survival function given in equation (4) is

$$
\mathrm{g}(\mathrm{x}, \alpha, \beta)=\frac{(1-\alpha) \beta \alpha^{\beta} \mathrm{f}(\mathrm{x})}{\left(1-\alpha^{\beta}\right)\{\mathrm{F}(\mathrm{x})+\alpha \overline{\mathrm{F}}(\mathrm{x})\}^{\beta+1}}
$$

Recently, several authors have used this approach to introduce new distributions. Jayakumar \& Sankaran ${ }^{10}$ defined a generalized uniform distribution using the approach of Nadarajah et al., ${ }^{8} \mathrm{Babu}^{11}$ introduced Weibull truncated negative binomial distribution. Further, Jayakumar \& Sankaran ${ }^{12}$ introduced generalized exponential truncated negative binomial distribution and studied its properties. Also, Jose \& Sivadas ${ }^{13}$ used the family given by equation (4) to introduce the negative binomial Marshall-Olkin Rayleigh distribution.

The contents of this paper are organized as follows. Section 2 deals with a generalized Rayleigh-truncated negative binomial distribution and its properties. Section 3 gives a real-life application. The concluding remarks are given in Section 4.

\section{Generalized Rayleigh-truncated negative binomial distribution}

In this section, we are focused on generalized Rayleigh truncated negative binomial (GR-TNB) distribution introduced by Jiju \& Lishamol. ${ }^{1}$ The distribution is derived by using generator approach of 
Nadarajah et al., ${ }^{8}$ They have examined various statistical properties of this distribution including estimation of parameters and have showed that this distribution is more flexible comparing to other generalizations of the rayleigh distribution. The survival function of the GR-TNB distribution is given by

$$
\left.\overline{\mathrm{G}}(\mathrm{x}, \alpha, \beta, \lambda, \theta)=\frac{\alpha^{\beta}}{1-\alpha^{\beta}}\left\{\gamma\left(\lambda+1,(\mathrm{x} / \theta)^{2}\right)+\overline{\alpha \gamma}\left(\lambda+1,(\mathrm{x} / \theta)^{2}\right)\right]^{-\beta}-1\right\}, \mathrm{x}, \alpha, \beta, \theta>0, \lambda>-1
$$

corresponding pdf is

$$
\mathrm{g}(\mathrm{x}, \alpha, \beta, \lambda, \theta)=\frac{(1-\alpha) \beta \alpha^{\beta} 2 \theta^{-2(\lambda+1)} \mathrm{x}^{2 \lambda+1} \mathrm{e}^{-(\mathrm{x} / \theta)^{2}}}{\Gamma(\lambda+1)\left(1-\alpha^{\beta}\right)\left\{\gamma\left(\lambda+1,(\mathrm{x} / \theta)^{2}\right)+\alpha \bar{\gamma}\left(\lambda+1,(\mathrm{x} / \theta)^{2}\right)\right\}^{\beta+1}} \mathrm{x}, \alpha, \beta, \theta>0, \lambda>-1
$$

and the hazard rate function (hrf) of the GR-TNB distribution becomes

$\mathrm{h}(\mathrm{x}, \alpha, \beta, \lambda, \theta)=\frac{(1-\alpha) \beta 2 \theta^{-2 \lambda-2} \mathrm{x}^{2 \lambda+1} \mathrm{e}^{-(\mathrm{x} / \theta)^{2}}\left[\gamma\left(\lambda+1, \frac{\mathrm{x}^{2}}{\theta^{2}}\right)+\alpha \bar{\gamma}\left(\lambda+1, \frac{\mathrm{x}^{2}}{\theta^{2}}\right)\right]^{-1}}{\Gamma(\lambda+1)\left(1-\left[\gamma\left(\lambda+1,(\mathrm{x} / \theta)^{2}\right)+\alpha \bar{\gamma}\left(\lambda+1,(\mathrm{x} / \theta)^{2}\right)\right]^{\beta}\right)}$

The GR-TNB distribution enfolds some sub models such as MOE GR distribution, MOE half normal distribution and MOE Reyleigh distribution. Figure 1 shows the plots for pdf and hrf for GR-TNB distribution with various parameter values. As seen in Figure 1, the pdf and the hrf of the GR-TNB distribution have several different shapes according to the values of the parameters. This shows that GR-TNB distribution is more flexible than Rayleigh distribution. The quantile

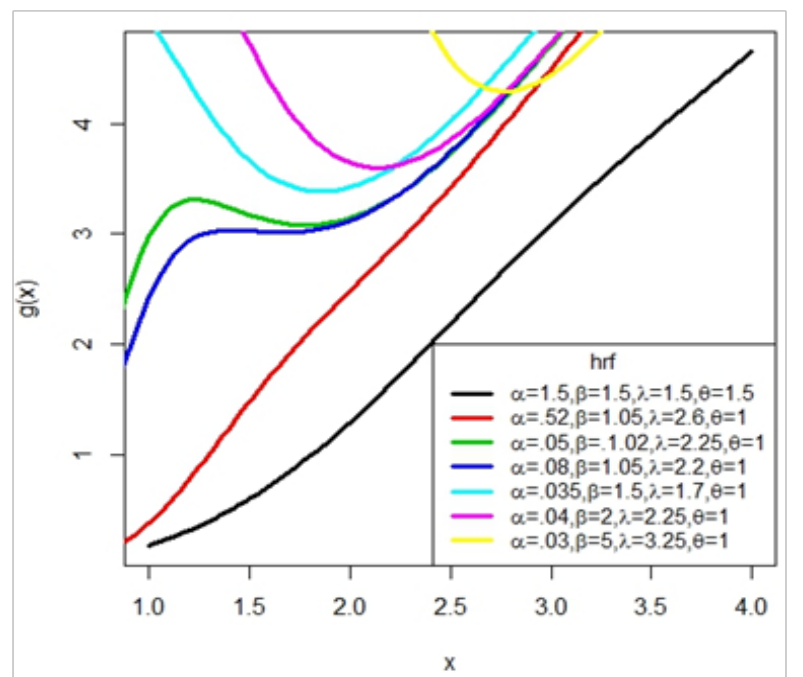

function of $\mathrm{X}$ follows GR-TNB distribution, it can be expressed as

$$
\mathrm{Q}(\mathrm{u})=\sqrt{\theta \mathrm{Q}_{\mathrm{G}}\left[\frac{1}{1-\alpha}\left[\left\{\frac{(1-\mathrm{u})\left(1-\alpha^{\beta}\right)}{\alpha^{\beta}}\right\}^{-\frac{1}{\beta}}+1\right]-\alpha\right]}
$$

where $\mathrm{u}$ is generated from the uniform $(0,1)$ distribution and $\mathrm{Q}_{\mathrm{G}}(\cdot)$ is the (standardized) gamma quantile function with shape parameter $\lambda+1$ and unit scale parameter (Figure 1).

\section{Simulation study}

In this section, we carry out Monte Carlo simulation study to assess the performance of the maximum likelihood estimates (MLE). The results are obtained from generating 1000 samples from the GRTNB distribution. For each replication, a random sample of size $\mathrm{n}=$ 50,100 and 200 is drawn from the GR-TNB distribution. The GRTNB random number generation was performed using the quantile function of GR-TNB distribution and the parameters are estimated by using the method of MLE by using package nlm in R, we get MLEs $\hat{\beta}, \hat{\theta}$ and $\hat{\lambda}$ for fixed $\alpha=0.2$ or $\alpha=0.02$. The evaluation of the performance is based on the bias and the mean squared errors (MSE) defined as follows:

Average bias of the simulated $\mathrm{N}$ estimates of $\mathrm{R}$ :

$$
\frac{1}{\mathrm{~N}} \sum_{\mathrm{i}=1}^{\mathrm{N}}\left(\hat{\mathrm{R}}_{\mathrm{i}}-\mathrm{R}\right)
$$

Average Mean square error of the simulated $\mathrm{N}$ estimates of $\mathrm{R}$ :

$$
\frac{1}{\mathrm{~N}} \sum_{\mathrm{i}=1}^{\mathrm{N}}\left(\hat{\mathrm{R}}_{\mathrm{i}}-\mathrm{R}\right)^{2}
$$

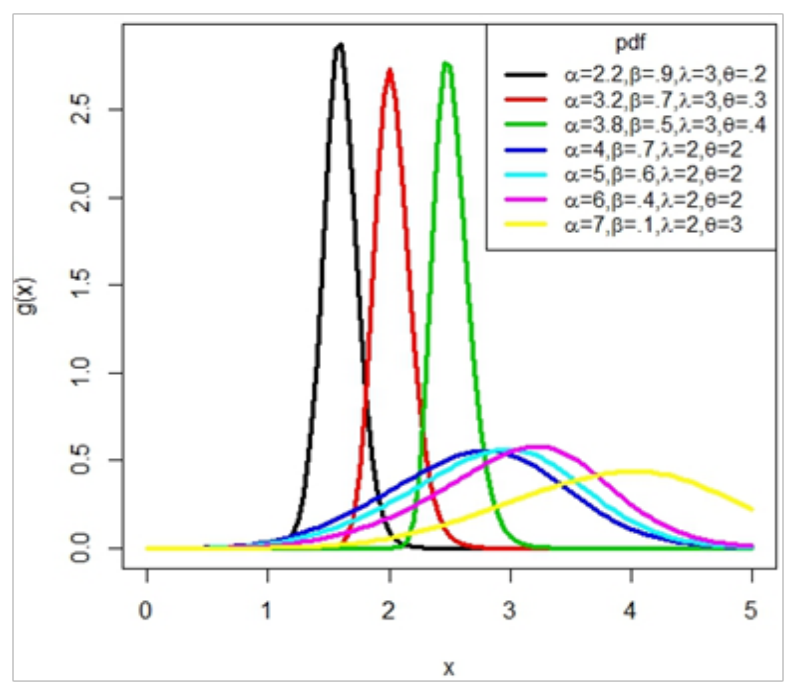

Figure I Graphs of pdf and hrf the GR-TNB distribution for different values of $\alpha, \beta, \lambda$ and $\theta$.

where $\mathrm{R}$ is the true value of parameters $\beta, \theta$ and $\lambda$ and also $\mathrm{N}$ is the number of replications. The initial values of parameter are $\beta=1.5, \theta=0.1$ and $\lambda=0.2$. The results of our simulation study are summarized. From this table, we can see that the bias and MSE of the MLEs converge to zero when the sample size is increased (Table 1).

\section{Data analysis}

In this section, we consider a real data set on breaking stress of carbon fibres of $50 \mathrm{~mm}$ length $(\mathrm{GPa})$ to assess the flexibility of the GR-TNB distribution over some well-known generalizations of Rayleigh distribution. The data have been previously used by Nichols $\&$ Padgettcit. ${ }^{14}$ The data are as follows: 
$0.39,0.85,1.08,1.25,1.47,1.57,1.61,1.61,1.69,1.80,1.84,1.87$ $1.89,2.03,2.03,2.05,2.12,2.35,2.41,2.43,2.48,2.50,2.53,2.55$, $2.55,2.56,2.59,2.67,2.73,2.74,2.79,2.81,2.82,2.85,2.87,2.88$, $2.93,2.95,2.96,2.97,3.09,3.11,3.11,3.15,3.15,3.19,3.22,3.22$, $3.27,3.28,3.31,3.31,3.33,3.39,3.39,3.56,3.60,3.65,3.68,3.70$, $3.75,4.20,4.38,4.42,4.70,4.90$

Table I Simulation Study for GR-TNB(x; $\alpha, \beta, \lambda, \theta)$ with $\alpha=0.2$ and $\alpha=0.02$

\begin{tabular}{|c|c|c|c|c|c|}
\hline \multirow[b]{2}{*}{$\mathbf{n}$} & \multirow[b]{2}{*}{ Parameters } & \multicolumn{2}{|c|}{$\alpha=0.2$} & \multicolumn{2}{|c|}{$\alpha=0.02$} \\
\hline & & Bias & MSE & Bias & MSE \\
\hline & $\beta$ & 0.047 & 0.2362 & 0.055 & 0.99 \\
\hline \multirow[t]{3}{*}{$n=50$} & $\theta$ & 0.098 & 0.3756 & 0.098 & 0.009 \\
\hline & $\lambda$ & 0.018 & 0.0391 & 0.092 & 0.003 \\
\hline & $\beta$ & 0.008 & 0.0001 & 0.004 & 0.099 \\
\hline \multirow[t]{3}{*}{$n=100$} & $\theta$ & 0.081 & 0.0098 & 0.009 & 0.009 \\
\hline & $\lambda$ & 0.005 & 0.0005 & 0.039 & 0.0009 \\
\hline & $\beta$ & 0.004 & 0.0003 & 0.001 & 0.002 \\
\hline \multirow[t]{2}{*}{$n=200$} & $\theta$ & 0.018 & 0.009 & 0.005 & 0.0001 \\
\hline & $\lambda$ & 0.0007 & 0.00006 & 0.009 & 0.000083 \\
\hline
\end{tabular}

We compare the results of GR-TNB distribution with following four-parameter generalizations of Rayleigh distribution which are generalized by using different generators:

1. Beta exponential generalized Rayleigh (BExpGR) distribution by Alzaatreh et al. ${ }^{15}$

2. Beta extended generalized Rayleigh (BEGR) distribution by Cordeiro et al. ${ }^{16}$

3. Exponentiated Kumaraswamy generalized Rayleigh (EKGR) distribution Lemonte et al. ${ }^{17}$

4. Extended beta generalized Rayleigh (EBGR) distribution Alexander et al. ${ }^{18}$

For each distribution, we estimated the unknown parameters (by the maximum likelihood method), the values of the - log-likelihood $(-\log \mathrm{L})$, AIC (Akaike Information Criterion), BIC (Bayesian Information Criterion), the values of the Kolmogorov-Smirnov (KS) statistic and the corresponding p-values. The result of comparison of the proposed distributions for these data. From these results we can see that GR-TNB distribution provide smallest - $\log$ L, AIC, BIC and $\mathrm{K}-\mathrm{S}$ statistics values and highest $\mathrm{p}$-value as compare to other distributions. This strongly claims that the proposed GR-TNB model provides better fit to the concerned data than the other distributions. Therefore, the GR-TNB distribution is an attractive alternative to the other available four-parameter generalized Rayleigh models in the literature (Table 2).

Table 2 Estimated values, logL, AIC, BIC, K-S statistics and-value for data set

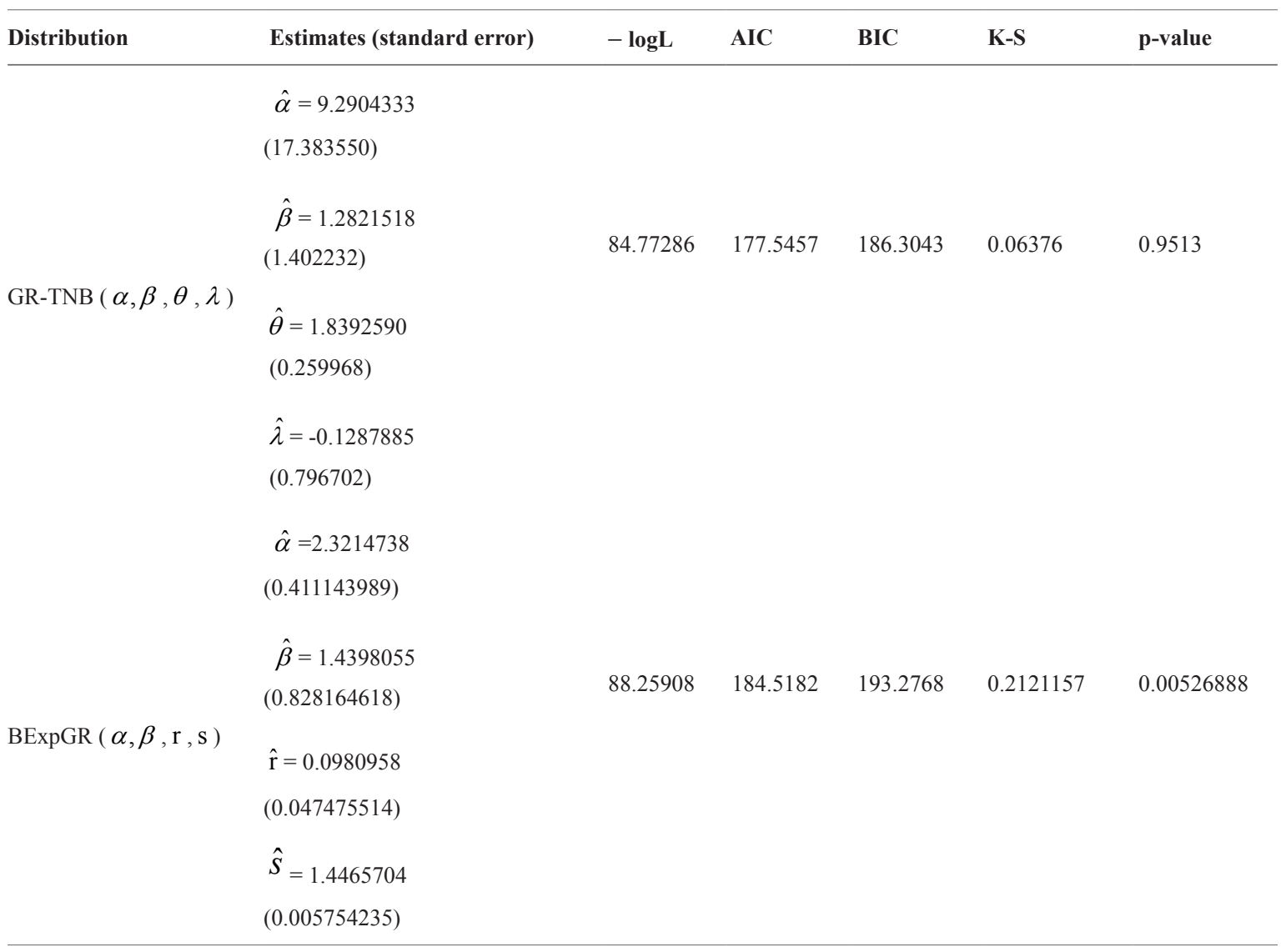




\begin{tabular}{|c|c|c|c|c|c|c|}
\hline Distribution & Estimates (standard error) & $-\log L$ & AIC & BIC & K-S & p-value \\
\hline $\operatorname{BEGR}(\alpha, \beta, \mathrm{r}, \mathrm{s})$ & $\begin{array}{l}\hat{\alpha}=2.410055279 \\
(0.405482144) \\
\hat{\beta}=6.3950288 \\
(9.68416177) \\
\hat{r}=4.833964992 \\
(6.914385767) \\
\hat{\mathrm{s}}=0.008840908 \\
(0.004205926)\end{array}$ & 88.47258 & 184.9452 & 193.7038 & 0.1154599 & 0.3424505 \\
\hline $\operatorname{EKGR}(\alpha, \beta, \mathrm{r}, \mathrm{s})$ & $\begin{array}{l}\hat{\alpha}=0.009924694 \\
(0.02485053) \\
\hat{\beta}=0.205804483 \\
(0.02541743) \\
\hat{\mathrm{r}}=9.787248978 \\
(7.00657360) \\
\hat{\mathrm{s}}=1.126722092 \\
(0.04244422)\end{array}$ & 85.75583 & 179.5117 & 188.2703 & 0.08173829 & 0.7699466 \\
\hline $\operatorname{EBGR}(\alpha, \beta, \mathrm{r}, \mathrm{s})$ & $\begin{array}{l}\hat{\alpha}=0.45962398 \\
(0.47172504) \\
\hat{\beta}=11.07897095 \\
(22.78252971) \\
\hat{\mathrm{r}}=3.5728010 \\
(3.7261659) \\
\hat{\mathrm{s}}=0.05118239 \\
(0.07335831)\end{array}$ & 188.5795 & 179.8209 & 188.5795 & 0.0810696 & 0.7785533 \\
\hline
\end{tabular}

\section{Conclusions}

In the last two decades, generalization approaches were adopted and practiced by many statisticians. In this study, we concentrated on such a generalization of Rayleigh distribution and presented a simulation study for verifying the validity of its estimates. A data set is used to prove the performance of GR-TNB distribution. The results present that the GR-TNB distribution provides better fits than existing distributions.

\section{Acknowledgements}

The second author is grateful to the Department of Science and Technology (DST), Govt. of India for the financial support under the INSPIRE Fellowship.

\section{Conflicts of interests}

Author declares that there are no conflicts of interest.

\section{References}

1. Jiju G, Lishamol T, A New Life Time Model: The Generalized Rayleigh-Truncated Negative Binomial Distribution. International Journal of Scientific Research in Mathematical and Statistical Sciences. 2018;5(6):1-12.

2. Rayleigh L. On the stability or instability of certain fluid motions. Proceedings of London Mathematical Society. 1880;1(1):57-70.

3. VG Vode. Inferential procedures on a generalized Rayleigh variate (I). Aplikace Matematiky. 1976;21(6):395-412. 
4. VG Vode. Inferential procedures on a generalized Rayleigh variate (II) Aplikace Matematiky. 1976;21(6):413-419.

5. GM Cordeiro, CT Cristino, EM Hashimoto, et al. The beta generalized Rayleigh distribution with applications to lifetime data. Statistical Papers. 2013;54(1):113-161.

6. AE Gomes, CQ Da-Silva, GM Cordeiro, et al. A new lifetime model: The Kumaraswamy generalized Rayleigh distribution. Journal of Statistical Computation and Simulation. 2014;84(2):290-309.

7. SMTK MirMostafaee, M Mahdizadeh, AJ Lemonte. The Marshall-Olkin extended generalized Rayleigh distribution: Properties and applications. Journal of Statistical Computation and Simulation. 2017;46(2):653-671.

8. S Nadarajah, K Jayakumar, MM Risti. A new family of lifetime models Journal of Statistical Computation and Simulation. 2012;83(8):1-16.

9. AW Marshall, I Olkin. A new method for adding a parameter to a family of distributions with application to the exponential and Weibull families Biometrica. 1997;84(3)641-652.

10. K Jayakumar, KK Sankaran. On a generalization of Uniform distribution and its Properties. Statistica. 2016;6(3):83-91.

11. MG Babu. On a generalization of Weibull distribution and its applications International Journal of Statistics and Applications. 2016;6(2):168-176.
12. K Jayakumar, KK Sankaran. Generalized Exponential Truncated Negative Binomial distribution. American Journal of Mathematical and Management Sciences. 2017;36(2):98-111.

13. KK Jose, R Sivadas. Negative binomial Marshall-Olkin Rayleigh distribution and its applications. Economic Quality Control. 2015;30(2):89-98.

14. Nichols MD, WJ Padgett. A bootstrap control chart for Weibull percentiles. Quality and Reliability Engineering. 2006;22(2):141-151.

15. Alzaatreh A, Lee C, Famoye F. A New Method for Generating Families of Continuous Distributions. Metron. 2013;(71):63-79.

16. Cordeiro GM, Ortega EMM, Silva G. The Beta Extended Weibull Family. Journal of Probability and Statistical Science. 2012;10:15-40.

17. Lemonte AJ, Barreto-Souza W, Cordeiro GM. The Exponentiated Kumaraswamy Distribution and Its Log-Transform. Brazilian Journal of Probability and Statistics. 2013;27(1):31-53.

18. Alexander C, Cordeiro GM, Ortega EMM, et al. Generalized BetaGenerated Distributions. Computational Statistics and Data Analysis. 2012;56:1880-1897. 